\title{
Crystal and magnetic structures of the oxide sulfides
}

\section{CaCoSO and BaCoSO}

\author{
Edward J. T. Salter, ${ }^{1}$ Jack N. Blandy, ${ }^{1,2}$ and Simon J. Clarke ${ }^{1, *}$ \\ ${ }^{1}$ Department of Chemistry, University of Oxford, Inorganic Chemistry \\ Laboratory, South Parks Road, Oxford, OX1 3QR, UK \\ ${ }^{2}$ Diamond Light Source Ltd., Harwell Science and Innovation Campus, \\ Didcot, OX11 ODE, UK.
}




\begin{abstract}
$\mathrm{CaCoSO}$, synthesised from $\mathrm{CaO}$, $\mathrm{Co}$ and $\mathrm{S}$ at $900{ }^{\circ} \mathrm{C}$ is isostructural with $\mathrm{CaZnSO}$ and $\mathrm{CaFeSO}$. The structure is non-centrosymmetric by virtue of the arrangement of the vertex-sharing $\mathrm{CoS}_{3} \mathrm{O}$ tetrahedra which are linked by their sulfide vertices to form layers. The crystal structure adopts space group $P 6_{3} m c$ (No. 186) and the lattice parameters are $a=3.7524(9) \AA$ and $c=11.138(3) \AA$ at room temperature with two formula units in the unit cell. The compound is highly insulating and powder neutron diffraction measurements reveal long range antiferromagnetic order with a propagation vector $\boldsymbol{k}=(1 / 3,1 / 3,1 / 2)$. The magnetic scattering from a powder sample can be modelled starting from a $120^{\circ}$ arrangement of $\mathrm{Co}^{2+}$ spin vectors in the triangular planes and then applying a canting out of the planes which can be modelled in the magnetic space group $C_{c} c$ (space group 9.40 in the Belov, Neronova \& Smirnova (BNS) scheme) with $\mathrm{Co}^{2+}$ moments of 2.72(5) $\mu_{\mathrm{B}}$. The antiferromagnetic structure of the recently-reported compound $\mathrm{BaCoSO}$ which has a very different crystal structure from $\mathrm{CaCoSO}$ is also described and this magnetic structure and the magnitude of the ordered moment $\left(2.75(2) \mu_{\mathrm{B}}\right)$ are found by experiment to be similar to those predicted computationally.
\end{abstract}




\section{Introduction}

Oxide chalcogenides are a class of compound in which ordering of the two different anions: $\mathrm{O}^{2-}$ and the larger and more polarisable $\mathrm{S}^{2-}, \mathrm{Se}^{2-}$, or $\mathrm{Te}^{2-}$ results in layered structures with segregation of metal cations according to their chemical preferences. These compounds have been of considerable interest as possible transparent $p$-type transparent conductors, ${ }^{1}$ and the investigation of related mixed-anion compounds led to the unexpected discovery of superconductivity in appropriately substituted lanthanide iron oxide arsenides. ${ }^{2}$ In turn, the investigation of the iron-based arsenide and chalcogenide superconductors has led to renewed exploration of oxide chalcogenides. The range of structure types and some of the properties of these compounds have recently been reviewed. ${ }^{3} \mathrm{We}$ and others reported previously ${ }^{4-6}$ the discovery of new structure types for oxide sulfides in the white wide-band-gap insulators containing alkaline earth and zinc cations. The non-centrosymmetric polar oxide sulfide $\mathrm{CaZnSO}$ with the structure shown in Figure 1(a) adopts a layered structure in which $\left[\mathrm{ZnS}_{3} \mathrm{O}\right]$ tetrahedra share all three basal sulfide vertices with other tetrahedra to form ${ }_{\infty}^{2}\left[\mathrm{ZnS}_{3 / 3} \mathrm{O}\right]$ layers and the tetrahedra are all oriented along the $c$ axis resulting an the non-centrosymmetric space group $P 6_{3} m c .{ }^{4,5}$ When the alkaline earth cation is the much larger $\mathrm{Ba}^{2+}$ a different structure is adopted for $\mathrm{BaZnSO}$ (Figure $1(\mathrm{~b})$ ) in which $\left[\mathrm{ZnS}_{2} \mathrm{O}_{2}\right]$ tetrahedra are linked by sharing each vertex with another tetrahedron. ${ }^{6}$ A previous report ${ }^{7}$ on the existence of $\mathrm{CaFeSO}$ has recently been augmented by further reports characterising this magnetic Mott-Hubbard insulating compound, ${ }^{8,9}$ which is isostructural with $\mathrm{CaZnSO}$. BaCoSO,${ }^{10}$ isostructural with BaZnSO, has also been described. Here we characterise a third member with the CaZnSO structure: $\mathrm{CaCoSO}$, and we make comparisons of the crystal structures and the magnetic ordering in this series of compounds. We also report the magnetic structure of $\mathrm{BaCoSO}$ and show that it is as predicted by recent computational investigations. ${ }^{10}$ 
(a)

(b)

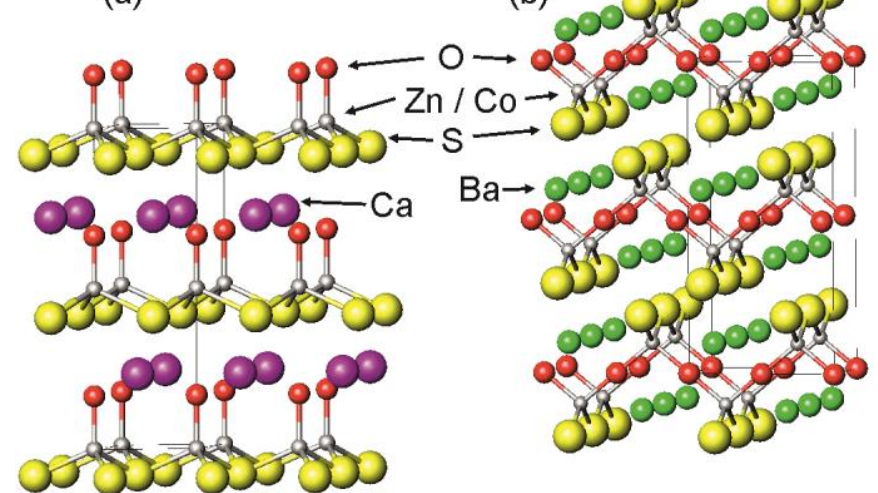

Figure 1. Crystal structures of (a) $\mathrm{Ca} M \mathrm{SO}\left(M=\mathrm{Fe},{ }^{8,9} \mathrm{Co}, \mathrm{Zn}^{4,5}\right)$ and (b) $\mathrm{Ba} M S O(M=$ Co, $\left.{ }^{10} \mathrm{Zn}^{6}\right)$

\section{Experimental.}

Synthesis. Because of the air sensitivity of some of the reactants, solids were handled in an argon-filled glove box. Both compounds were synthesized from stoichiometric amounts of alkaline earth oxides and elemental $\mathrm{Co}$ and $\mathrm{S}$ with the ground mixtures contained in alumina crucibles within dried evacuated silica tubes. In both cases the initial heating stage was performed at $2{ }^{\circ} \mathrm{C} \mathrm{min}^{-1}$ to enable elemental sulfur to react without generating a large over-pressure. $\mathrm{CaCoSO}$ was synthesised on the $4 \mathrm{~g}$ scale using $\mathrm{CaO}$ (obtained from the thermal decomposition of $\mathrm{CaCO}_{3}$ (ALFA 99.99\%)), Co (ALFA 99.99\%) and S (ALFA 99.9995\%). The reaction was heated at $900{ }^{\circ} \mathrm{C}$ for two periods of two days with an intermediate regrinding and this resulted in material that was free of impurities as judged by laboratory X-ray powder diffraction (XRPD). Lower reaction temperatures resulted in impure products. BaCoSO was synthesised on a similar scale according to the method described in ref. 10. $\mathrm{BaO}$ (Aldrich 99.99\%), Co (Alfa Aesar 99.998\% ) and S (Alfa Aesar 99.999\%) were ground together, pressed into a pellet $\left(300 \mathrm{MPa}\right.$ ) and heated at $900^{\circ} \mathrm{C}$ for $12 \mathrm{~h}$ (after heating at $2{ }^{\circ} \mathrm{C} \mathrm{min}{ }^{-1}$ ). This product was reground, repelletized and heated at $940{ }^{\circ} \mathrm{C}$ for 100 hours.

Diffraction measurements. Laboratory XRPD patterns to monitor the progress of reactions and for initial structural characterisation were measured using a PANAlytical X'-Pert diffractometer with $\mathrm{Cu}-K \alpha_{1} / \alpha_{2}$ radiation or with a PANAlytical Empyrean instrument with $\mathrm{Cu}-K \alpha_{1}$ radiation. Neutron powder diffraction (NPD) data for $\mathrm{CaCoSO}$ were collected at ambient temperature and at $8 \mathrm{~K}$ on a $4 \mathrm{~g}$ sample using 
the now-retired diffractometer D1A at the ILL Grenoble. Data for BaCoSO were collected at ambient temperature and $3.5 \mathrm{~K}$ using a $4.5 \mathrm{~g}$ sample using the diffractometer D2B at ILL. The samples were contained in thin-walled vanadium cylinders. For the low temperature measurements the samples were cooled using different closed-cycle refrigerators. Refinements against the diffraction data were carried out using Topas Academic V $5^{11}$ and magnetic scattering was modelled by introducing additional magnetic-only phases to the refinements. Magnetic models were deduced with the aid of the ISODISTORT software suite. ${ }^{12}$

Physical property measurements. Magnetic susceptibility measurements for CaCoSO were made using a Quantum Design MPMS-5 SQUID magnetometer. 4probe resistivity measurements on a sintered pellet of $\mathrm{CaCoSO}$ were attempted using a home-built apparatus.

\section{Results and Discussion.}

\section{Structure, properties and magnetic order of CaCoSO.}

Analysis of the XRPD pattern of CaCoSO revealed that the phase was not contaminated by significant amounts of impurity phases and that the phase was isostructural with $\mathrm{CaZnSO}$. The structure at ambient temperature was refined from the NPD pattern (Figure 2(a)) and small impurities: $\mathrm{CaO}$ (0.4\% by mass), $\mathrm{Co}_{9} \mathrm{~S}_{8}(3.2 \%)$ and $\mathrm{CaCO}_{3}$ (calcite; $0.2 \%$ ) were included in the refinement along with reflections from the vanadium sample holder. The refinement results at room temperature are presented in Table 1 and Table 2. Table 3 summarises the key structural parameters for the three isostructural compounds $\mathrm{CaZnSO}, \mathrm{CaFeSO}$ and $\mathrm{CaCoSO}$. 


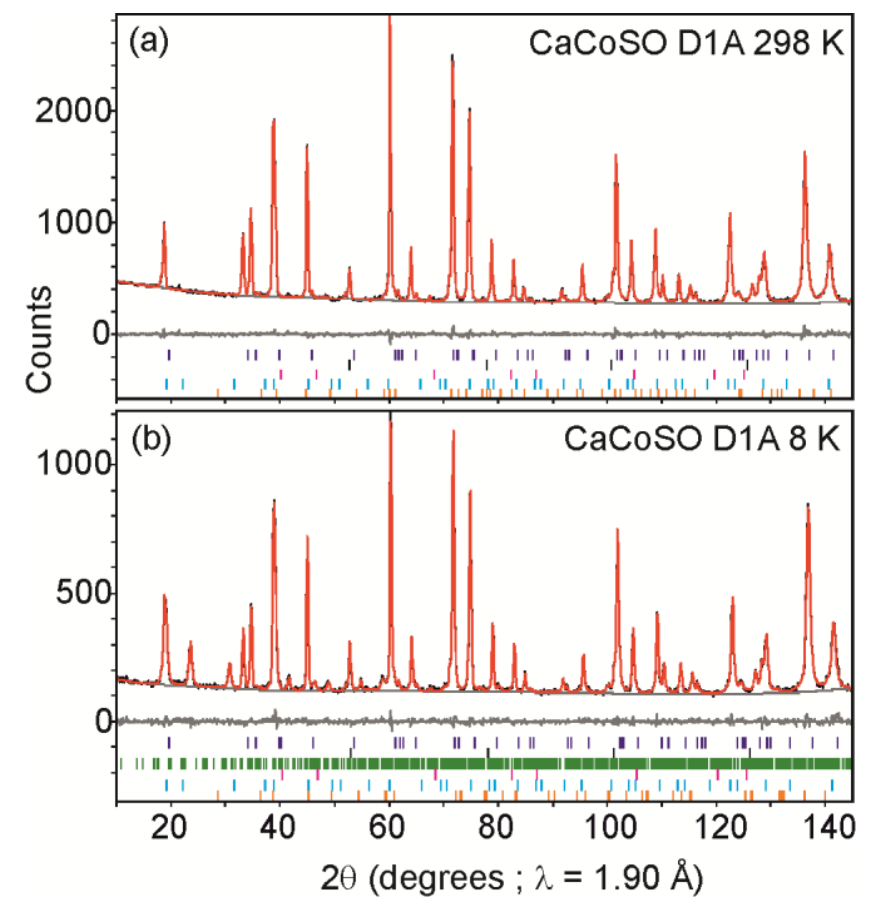

Figure 2. Rietveld refinement against NPD data for CaCoSO at (a) $298 \mathrm{~K}$ and (b) $8 \mathrm{~K}$. Data (black line), calculated (red), difference (gray) are shown and the background function (gray line) is also shown. Tick marks show reflection positions. From top to bottom these are for the CaCoSO nuclear phase (purple), vanadium sample holder (black), CaCoSO magnetic phase (green $-8 \mathrm{~K}$ data only), $\mathrm{CaO}$ ( $0.4 \%$ by mass) (magenta), $\mathrm{Co}_{9} \mathrm{~S}_{8}(3.2 \%)$ (cyan) and $\mathrm{CaCO}_{3}$ (calcite; $0.2 \%$ ) (orange).

Comparison of bond lengths and angles in the three compounds reveals a trend in the $M-\mathrm{O}$ and $M-\mathrm{S}$ distances from $M=\mathrm{Fe}$ to Co which reflects the normal trends across the series. In $\mathrm{CaZnSO}$ the ratio of the $\mathrm{Zn}-\mathrm{S}$ and $\mathrm{Zn}-\mathrm{O}$ distances is similar to that calculated from the tables of published ionic radii, ${ }^{13}$ while for both $\mathrm{CaFeSO}$ and $\mathrm{CaCoSO}$ the $M-\mathrm{O}$ distance is relatively short, consistent with relatively strong covalent bonding between the transition metal cations and oxide. A comparison of the $\mathrm{Co}-\mathrm{O}$ and $\mathrm{Co}-\mathrm{S}$ distances in $\mathrm{CaCoSO}$ with the values obtained for spinels, where $\mathrm{Co}^{2+}$ ions occupy regular tetrahedral sites, reveals that in $\mathrm{CaCoSO}$ the $\mathrm{Co}-\mathrm{O}$ distance is $4.3 \%$ shorter than that in $\mathrm{Co}_{3} \mathrm{O}_{4}{ }^{14}$ and the $\mathrm{Co}-\mathrm{S}$ distance is $1.7 \%$ longer than that in $\mathrm{Cr}_{2} \mathrm{CoS}_{4}{ }^{15}$ In $\mathrm{BaCoO}_{2}$, one of few representatives of oxides containing tetrahedral $\mathrm{Co}^{2+}$ ions and an electropositive metal cation, ${ }^{16}$ the mean $\mathrm{Co}-\mathrm{O}$ distance of $1.904 \AA$ is 2.4\% longer than in $\mathrm{CaCoSO}$, reflecting the relative shortness of the $\mathrm{Co}-\mathrm{O}$ distance in $\mathrm{CaCoSO}$. The data also reveal that of the three compounds, the angles in the $\mathrm{CoS}_{3} \mathrm{O}$ tetrahedron are the closest to the angles in a regular tetrahedron. The ligand field in $\mathrm{CaCoSO}$ is discussed below. 
Table 1. Refinement results for $\mathrm{CaCoSO}$ at room temperature

\begin{tabular}{|l|l|}
\hline Formula & CaCoSO \\
\hline Radiation & Neutrons $\lambda=1.901 \AA$ \\
\hline Instrument & D1A (ILL) \\
\hline Physical form & Black powder \\
\hline$T(\mathrm{~K})$ & 298 \\
\hline Crystal system & Hexagonal \\
\hline Space group & $P 6_{3} m c($ No. 186$)$ \\
\hline Formula weight & 147.1 \\
\hline$a(\AA)$ & $3.7415(8)$ \\
\hline$c(\AA)$ & $11.106(2)$ \\
\hline$V\left(\AA^{3}\right)$ & $134.64(6)$ \\
\hline$Z$ & 2 \\
\hline$\rho_{\text {calc }}\left(\mathrm{Mg} \mathrm{m}^{-3}\right)$ & $3.628(2)$ \\
\hline$\chi^{2}$ & 1.23 \\
\hline$w R_{p}$ & 0.0255 \\
\hline$R_{\text {Bragg }}$ & 0.0083 \\
\hline
\end{tabular}


Table 2. Refined atomic parameters at room temperature for $\mathrm{CaCoSO}$.

\begin{tabular}{|l|l|l|l|l|l|l|}
\hline Atom & Site & $x$ & $y$ & $z$ & $100 \times\left(U_{\text {iso }}\left(\AA^{2}\right)\right)$ & Occ $^{a}$ \\
\hline $\mathrm{Ca}$ & $2 b$ & $1 / 3$ & $2 / 3$ & $0.2682(3)$ & $0.59(5)$ & 1 \\
\hline $\mathrm{Co}$ & $2 a$ & 0 & 0 & $0^{*}$ & $0.66(8)$ & 1 \\
\hline $\mathrm{S}$ & $2 b$ & $2 / 3$ & $1 / 3$ & $0.0789(4)$ & $0.50(8)$ & 1 \\
\hline $\mathrm{O}$ & $2 a$ & 0 & 0 & $0.3326(3)$ & $0.66(3)$ & 1 \\
\hline
\end{tabular}

$* z$ coordinate for Co fixed at 0 in this non-centrosymmetric space group $P 6_{3} m c$.

${ }^{a}$ Not refined

Table 3. Selected bond lengths $(\AA)$ and angles (degrees) for CaCoSO compared with literature values for $\mathrm{CaFeSO}^{8}$ and $\mathrm{CaZnSO}^{5}$

\begin{tabular}{|l|l|l|l|}
\hline Bond or angle & $\mathrm{CaFeSO}^{8}$ & $\mathrm{CaCoSO}$ & $\mathrm{CaZnSO}^{5}$ \\
\hline $\mathrm{Ca}-\mathrm{O}[3](\AA)$ & $2.298(4)$ & $2.276(2)$ & $2.2857(3)$ \\
\hline $\mathrm{Ca}-\mathrm{S}[3](\AA)$ & $2.982(9)$ & $3.014(4)$ & $3.0346(8)$ \\
\hline $\mathrm{M}-\mathrm{O}[1](\AA)$ & $1.88(1)$ & $1.859(3)$ & $1.8996(5)$ \\
\hline $\mathrm{M}-\mathrm{S}[3](\AA)$ & $2.391(6)$ & $2.331(2)$ & $2.3716(5)$ \\
\hline & & & \\
\hline $\mathrm{M}-\mathrm{S} / \mathrm{M}-\mathrm{O}$ & $1.27(1)$ & $1.254(3)$ & $1.248(1)$ \\
\hline $\mathrm{O}-\mathrm{M}-\mathrm{S}[3]\left(^{\circ}\right)$ & $114.76(4)$ & $112.1(1)$ & $113.84(3)$ \\
\hline $\mathrm{S}-\mathrm{M}-\mathrm{S}[3]\left(^{\circ}\right)$ & $103.70(3)$ & $106.8(1)$ & $104.77(3)$ \\
\hline
\end{tabular}

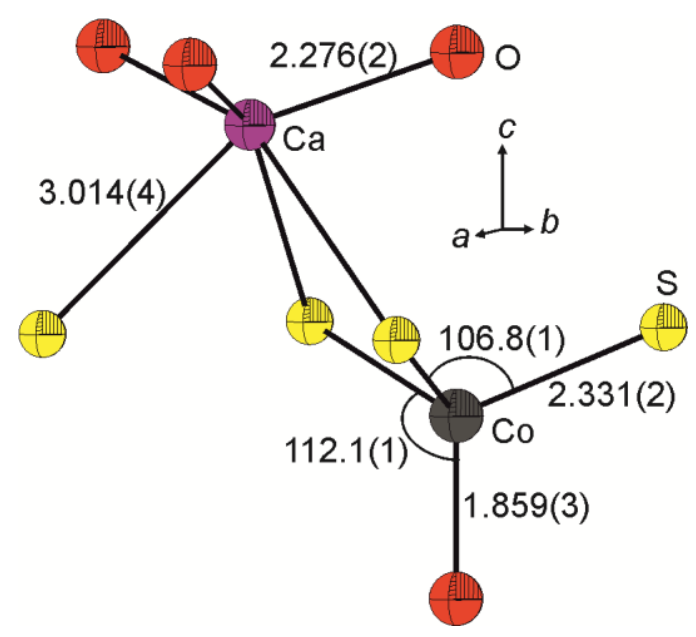

Figure 3. Structure of CaCoSO showing isotropic displacement ellipsoids (at the $99 \%$ level) and selected bond lengths (in $\AA$ ) and angles (in degrees). 


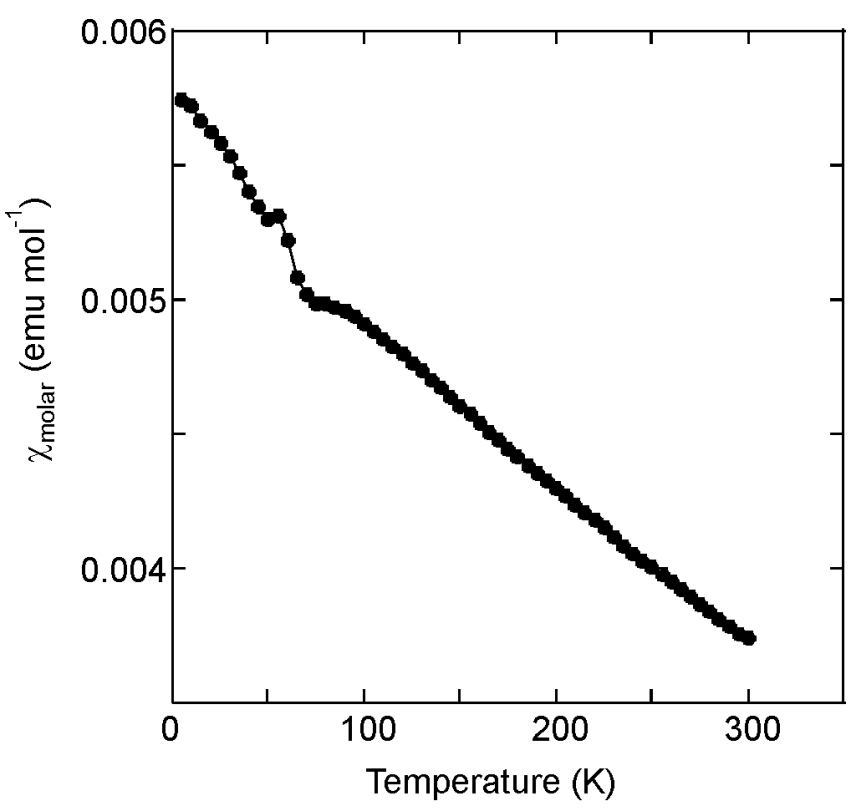

Figure 4. The magnetic susceptibility of CaCoSO as a function of temperature.

The magnetic susceptibility of $\mathrm{CaCoSO}$ is shown in Figure 4. A measurement of magnetisation against applied field at room temperature was non-linear suggesting a minuscule (below the detection limits of the diffraction experiments) ferromagnetic impurity was present. The magnetic susceptibility was therefore obtained by making measurements as a function of temperature at $3 \mathrm{~T}$ and $4 \mathrm{~T}$, fields above the saturation field of the impurity, and obtaining the temperature dependence of the susceptibility from the differences between the values measured at the two fields. The sample was cooled in zero applied field. The magnetic susceptibility reveals a kink at around $80 \mathrm{~K}$ which we presume indicates the onset of magnetic order, and there is a further feature at around $60 \mathrm{~K}$. The sample did not obey the Curie-Weiss law at high temperatures: a plot of the inverse susceptibility against temperature in the range $200-300 \mathrm{~K}$ superficially appeared linear, but the intercept with the temperature axis (i.e. the value of the Weiss Temperature) was about $-500 \mathrm{~K}$, so any attempt to extract a reliable effective magnetic moment for $\mathrm{Co}^{2+}$ was not justified. The sample was found to be highly insulating, and the resistance of the pellet was too high to enable a value for the resistivity to be extracted. This is consistent with localization of $\mathrm{Co}^{2+}$ electrons.

At $8 \mathrm{~K}$ new reflections are evident in the NPD pattern of CaCoSO (Figure 2(b)) which are consistent with magnetic ordering. The positions of the magnetic reflections are 
clearly different from those reported for CaFeSO ${ }^{9}$ Analysis of these new reflections using the ISODISTORT software ${ }^{12}$ revealed that their positions could be accounted for by a magnetic structure with a propagation vector, $k=(1 / 3,1 / 3,1 / 2)$. Analysis of possible distortion modes that would account for the intensities of these reflections showed that most of the magnetic scattering could be accounted for by arranging the $\mathrm{Co}^{2+}$ moments, which describe a triangular lattice in the $a b$ plane, on a $120^{\circ}$ structure with the moments oriented within the triangular plane. This is one way to satisfy the inherent frustration between antiferromagnetic alignment of nearest neighbour spins and their arrangement on a triangular lattice. This model required a single parameter corresponding to the magnitude of the $\mathrm{Co}^{2+}$ moment. However some discrepancies remained in the intensities of the most intense magnetic reflections (See Supplementary Information, Figure S1). Introduction of an out-of-plane canting distortion of the moments enabled these discrepancies to be accounted for by the introduction of one further refined parameter to model the canting and with constraints applied to ensure that the $\mathrm{Co}^{2+}$ ions all carried equal moments. The intensity discrepancies were only accounted for by a canting in which the moments within a single triangular Co layer were not all canted in the same sense. This resulted in the loss of three-fold rotational symmetry and so the magnetic model shown in Figure 5 is monoclinic and is described in the magnetic space group $C_{c} c$ (space group 9.40 in the Belov, Neronova \& Smirnova (BNS) scheme which is a setting of space group $C_{2 c} m^{\prime}$ (8.6.43) in the Opechowski \& Guccione (OG) scheme). ${ }^{17}$ The ordered moment on $\mathrm{Co}^{2+}$ was refined to be $2.75(5) \mu_{\mathrm{B}}$. The fit to the NPD data at $8 \mathrm{~K}$ is shown in Figure 2(b) and full lists of refinement results are included as supplementary information (Tables S1 - S5). It should be noted that a powder neutron diffraction experiment places intrinsic limitations on the ability to unambiguously determine the relative moment directions, ${ }^{18}$ and several models were found which could account equally well for the observed magnetic intensities. These models were all rooted in the $120^{\circ}$ structure with an out-of-plane canting applied as described above, and we have chosen a magnetic model in which the moments carried by all the $\mathrm{Co}^{2+}$ ions have equal magnitudes because the nuclear model contains a single $\mathrm{Co}^{2+}$ ion. A single crystal neutron diffraction experiment could, in principle, be used to distinguish these models. In the refinements we retained the ambient temperature nuclear model at low 
temperature as there was no evidence, at the resolution of our instrument, for a structural distortion accompanying the magnetic ordering.

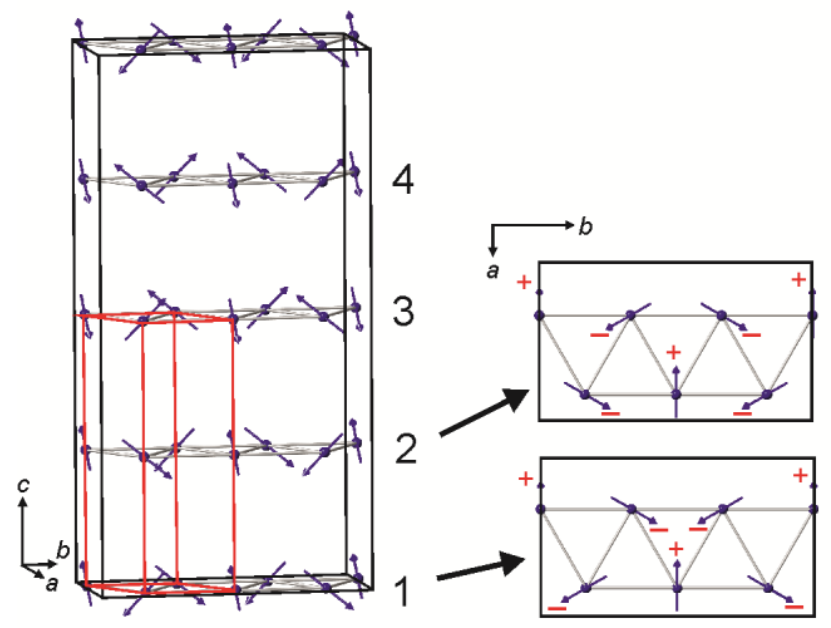

Figure 5. The magnetic model for CaCoSO derived from NPD data. The dimensions of the structural unit cell are depicted in red. Co atoms are shown. Other atoms are omitted for clarity. Tables S4 and S5 describe the model in detail. At right are shown the arrangements of Co moments in the layers labelled 1 and 2 viewed perpendicular to the layers. The canting (moments labelled " + " are canted to point above the plane, moments labelled "-" are canted to point below the plane) destroys the three-fold rotational symmetry. Layers 3 and 4 are aligned antiferromagnetically with respect to layers 1 and 2 respectively.

The $\mathrm{Co}^{2+}$ coordination environment has $\mathrm{C}_{3 v}$ symmetry and, as described above, the $\mathrm{Co}-\mathrm{O}$ distance is relatively short and the $\mathrm{Co}-\mathrm{S}$ bond is relatively long. There are several compounds in the bio-inorganic chemistry literature that have a similar distortion of the tetrahedral ligand field around the transition metal ion. An example that mimics well the $\mathrm{CaCoSO}$ case is the compound $\left[\mathrm{PhB}\left(\mathrm{CH}_{2} \mathrm{P}(i \mathrm{Pr})_{2}\right)_{3}\right] \mathrm{FeN}^{19}$ in which there is a strongly $\sigma$ and $\pi$ donating apical ligand (nitride) and $\sigma$-donor ligands (phosphine) describing the basal triangle. DFT calculations on $\left[\mathrm{PhB}\left(\mathrm{CH}_{2} \mathrm{P}(i \mathrm{Pr})_{2}\right)_{3}\right] \mathrm{FeN}$ quantify that in $\mathrm{C}_{3 v}$ symmetry the degenerate $d_{x z} / d_{y z}$ pair will lie highest in energy as they are $\pi$-antibonding with respect to the apical ligand and $\sigma$ antibonding with respect to the basal ligands. The degenerate $d_{x y} / d_{x 2-y 2}$ pair will lie low in energy. Calculations using a range of similar molecules with this distorted tetrahedral geometry ${ }^{20-23}$ show that the energy of $d_{z 2}$ is sharply dependent on the geometry and on the bonding characteristics of the ligands. Since in $\mathrm{CaCoSO}$ the distortion from tetrahedral is relatively small and is fairly similar to that in 
$\left[\mathrm{PhB}\left(\mathrm{CH}_{2} \mathrm{P}(i \mathrm{Pr})_{2}\right)_{3}\right] \mathrm{FeN},{ }^{19}$ we propose that the occupancy of the $d$ levels will be as calculated for that compound: $d_{z 2}$ will be comparable in energy to the higher-lying degenerate $d_{x z} / d_{y z}$ pair and the configuration will be $\left\{\left(d_{x y}\right)^{2}\left(d_{x 2-y 2}\right)^{2}\right\}\left\{\left(d_{x z}\right)^{1}\left(d_{y z}\right)^{1}\right\}\left(d_{z 2}\right)^{1}$. There will be three unpaired electrons and no first order orbital contribution to the moment. The refined value of the moment of $2.72(5) \mu_{\mathrm{B}}$ is consistent with this highspin scenario given that there will be considerable covalency which will lower the value of the ordered moment from the spin-only value of $3 \mu_{\mathrm{B}}$. Further measurements on single crystal samples would be required to further examine the exchange interactions between $\mathrm{Co}^{2+}$ moments to rationalise the magnetic structure in this system with a non-centrosymmetric nuclear structure.

$$
\begin{gathered}
x z \text { 手牛 } y z \\
\text { 手 } z^{2} \\
x y \text { 年柇 } x^{2}-y^{2}
\end{gathered}
$$

Figure 6. The proposed ligand field for $\mathrm{CaCoSO}$ based on analogous molecular systems.

\section{Magnetic Structure of BaCoSO}

The sample of BaCoSO contained some impurity phases ( $\mathrm{BaS}$ (4\% by mass) and $\mathrm{CoO}$ ( $2.4 \%$ by mass)) which were readily accounted for in the refinements. Refinement against D2B data at ambient temperature (Figure 7(a), Tables S6 - S7) confirmed the structural model reported by Valldor et al. in space group $\mathrm{Cmcm} .{ }^{10}$ At $3.5 \mathrm{~K}$ additional reflections were evident (Figure 7(b)) and the proposed antiferromagnetic model with the lowest computed energy reported by Valldor et al. ${ }^{10}$ and shown in Figure 8, together with the magnetic scattering from the $\mathrm{CoO}$ impurity, ${ }^{24}$ was able to account completely for the additional intensity. The magnetic model has a propagation vector $k=\left(\begin{array}{lll}1 / 2 & 0 & 0\end{array}\right)$ and may be described in space group $P_{c} m a 2$ (28.94 in the BNS scheme; a setting of space group $C_{p} m^{\prime} m 2^{\prime}(35.10 .245)$ in the OG scheme) as described in Table 4 and Tables S8 - S12. All four nearest neighbour couplings between $\mathrm{Co}^{2+}$ ions via $\mathrm{Co}-\mathrm{O}-\mathrm{Co}$ and $\mathrm{Co}-\mathrm{S}-\mathrm{Co}$ linkages are antiferromagnetic and the arrangement is unfrustrated. The refined moment on $\mathrm{Co}^{2+}$ ions in $\mathrm{BaCoSO}$ was 
2.75(2) $\mu_{\mathrm{B}}$, which is similar to the range of 2.6-2.8 $\mu \mathrm{B}$ per $\mathrm{Co}^{2+}$ ion estimated from the computation reported by Valldor et al. ${ }^{10}$ and similar to the value in CaCoSO.

Table 4. Summary of the parameters describing the magnetic symmetry and unit cell for BaCoSO.

\begin{tabular}{|l|l|}
\hline Magnetic Space group & $P_{c} m a 2(28.94)(\mathrm{BNS}$ scheme $)$ \\
\hline$a(\AA)$ & $6.07894(6)(c$ in nuclear structure $)$ \\
\hline$b(\AA)$ & $12.6976(1)$ \\
\hline$c(\AA)$ & $7.94770(8)(2 \times a$ in nuclear structure $)$ \\
\hline$V\left(\AA^{3}\right)$ & $613.47(1)$ \\
\hline
\end{tabular}

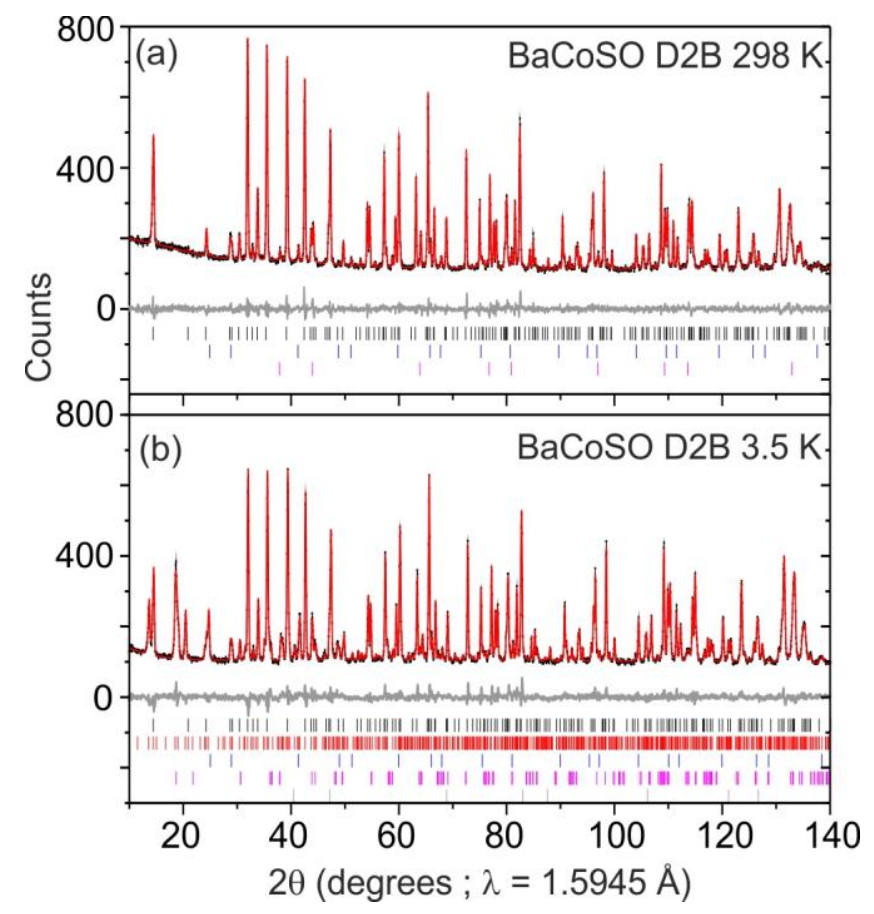

Figure 7. Refinements against NPD data for BaCoSO. (a) Room temperature (nuclear model only). (b) $3.5 \mathrm{~K}$ (nuclear and magnetic models). The refinements confirm the nuclear structure found by Valldor et al. ${ }^{10}$ and their magnetic structure proposed by computational methods. Full refinement details are given as supporting information. The figures show the data (black line), fit (red line) and difference (grey line) together with tick marks for the phases present: from top: BaCoSO nuclear (black), BaCoSO magnetic (red - $3.5 \mathrm{~K}$ data only), $\mathrm{BaS}$ ( $4 \%$ by mass - blue), $\mathrm{CoO}$ ( $2.4 \%$ - pink), Al sample environment (gray - $3.5 \mathrm{~K}$ data only). 


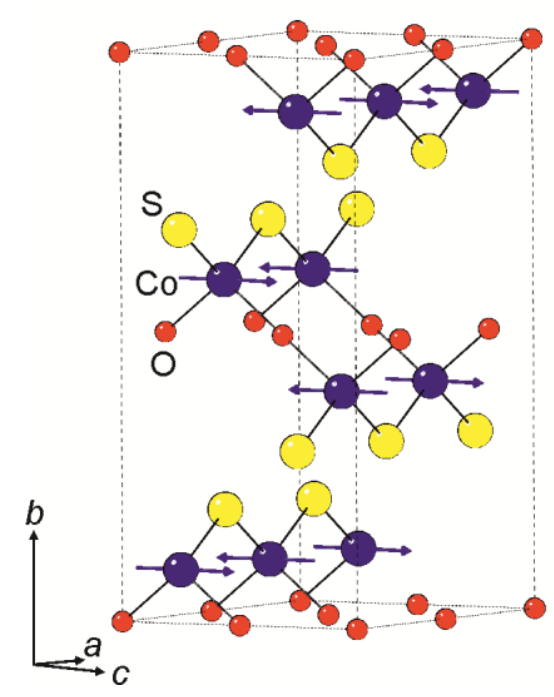

Figure 8. Magnetic structure of $\mathrm{BaCoSO}$ showing the antiferromagnetic arrangement of nearest neighbour moments via $\mathrm{Co}-\mathrm{O}-\mathrm{Co}$ and $\mathrm{Co}-\mathrm{S}-\mathrm{Co}$ linkages. $\mathrm{Ba}$ atoms are omitted for clarity. The unit cell vectors are depicted for the magnetic cell (see Table 4) which has double the volume of the structural unit cell (Figure S2).

\section{Conclusions.}

We have described a new non-centrosymmetric insulating antiferromagnet $\mathrm{CaCoSO}$ in which the frustration between the moments of high spin $\mathrm{Co}^{2+}$ ions spins inherent to the triangular arrangement is relieved by the adoption of a canted $120^{\circ}$ structure. We have verified that the unfrustrated magnetic structure of the isostoichiometric compound $\mathrm{BaCoSO}$, which has a different crystal structure, is that predicted from computation. In both compounds the ordered $\mathrm{Co}^{2+}$ moments are similar at about 2.7 $\mu_{\mathrm{B}}$, reflecting a largely quenched orbital moment and a reduction below the spin-only value due to covalency.

Acknowledgements. We thank Dr E. Suard, Dr D. R. Parker, Dr. C. F Smura and Dr. D. N. Woodruff for assistance on D1A and D2B and we thank the ILL for the award of beam time. We acknowledge the financial support of the EPSRC (Grant EP/E025447/1) and the Diamond Light Source (studentship support for JNB).

Supporting Information Available: Further tables of refined structural parameters.

Author Information. email: simon.clarke@chem.ox.ac.uk 


\section{References}

(1) Ueda, K.; Inoue, S.; Hirose, S.; Kawazoe, H.; Hosono, H. Appl. Phys. Lett. 2000, 77, 2701-2703.

(2) Kamihara, Y.; Watanabe, T.; Hirano, M.; Hosono, H. J. Am. Chem. Soc. 2008, 130, 3296-3297.

(3) Clarke, S. J.; Adamson, P.; Herkelrath, S. J. C.; Rutt, O. J.; Parker, D. R.; Pitcher, M. J.; Smura, C. F. Inorg. Chem. 2008, 47, 8473-8486.

(4) Petrova, S. A.; Marevich, V. P.; Zakharov, R. G.; Selivanov, E. N.; Chumarev, V. M.; Udoeva, L. Yu. Dokl. Chem. 2003, 393, 255-258.

(5) Sambrook, T.; Smura, C. F.; Clarke, S. J.; Ok, K. M.; Halasyamani, P. S. Inorg. Chem. 2007, 46, 2571-2574.

(6) Broadley, S.; Gál, Z. A.; Corà, F.; Smura, C. F.; Clarke, S. J. Inorg. Chem. 2005, 44, 9092-9096.

(7) Selivanov, E. N.; Chumarev, V. M.; Gulyaeva, R. I.; Marevich, V.P.; Vershinin, A. D.; Pankratov, A. A.; Korepanova, E. S. Inorg. Mater. 2004, 40, 845-850.

(8) Delacotte, C.; Pérez, O.; Pautrat, A.; Berthebaud, D.; Hébert, S.; Suard, E.;

Pelloquin, D.; Maignan, A. Inorg. Chem. 2015, 54, 6560-6565.

(9) Jin, S. F.; Huang, Q.; Lin, Z. P.; Li, Z. L.; Wu, X. Z.; Ying, T. P.; Wang, G.; Chen, X. L. Phys. Rev. B 2015, 91, 094420.

(10) Valldor, M.; Rössler, U. K.; Prots, Y.; Kuo, C. Y.; Chiang, J. C.; Hu, Z. W.; Pi, T. W.; Kniep, R.; Tjeng, L. H. Chem. Eur. J. 2015, 21, 10821-10828.

(11) Coelho, A. A. TOPAS Academic: General Profile and Structure Analysis Software for Powder Diffraction Data, Version 5 2015. http://www.topasacademic.net/.

(12) Campbell, B. J.; Stokes, H. T.; Tanner, D. E.; Hatch, D. M. J. Appl. Crystallogr. 2006, 39, 607-614.

(13) Shannon, R. D. Acta Crystallogr. A 1976, 32, 751.

(14) Picard, J. P.; Baud, G.; Besse, J. P.; Chevalier, R. J. Less-Common Met. 1980, 75, 99-104.

(15) Vaqueiro, P.; Sommer, S.; Powell, A. V. J. Mater. Chem., 2000, 10, 2381-2387.

(16) Spitsbergen, U.; Acta Crystallogr. 1960, 13, 197-198. 
(17) Litvin, D. B. Magnetic Group Tables: 1-, 2- and 3-Dimensional Magnetic

Subperiodic Groups and Magnetic Space Groups, International Union of

Crystallography 2013; ISBN 978-0-9553602-2-0 doi:10.1107/9780955360220001.

(18) Shirane, G. Acta Crystallogr. 1959, 12, 282-285.

(19) Betley, T. A.; Peters, J. C. J. Am. Chem. Soc. 2004, 126, 6252-6254.

(20) Mehn, M. P.; Peters, J. C. J. Inorg. Biochem. 2006, 100, 634-643.

(21) McSkimming, A.; Harman, W. H. J. Am. Chem. Soc. 2015, 137, 8940-8943.

(22) Brown, S. D.; Peters, J. C. J. Am. Chem. Soc. 2005, 127, 1913-1923.

(23) Hohenberger, J.; Ray, K.; Meyer, K. Nat. Commun. 2012, 3, 720.

(24) Jauch, W.; Reehuis, M.; Bleif, H. J.; Kubanek, F.; Pattison, R. Phys. Rev. B 2001, 64, 052102. 
For Table of Contents Only

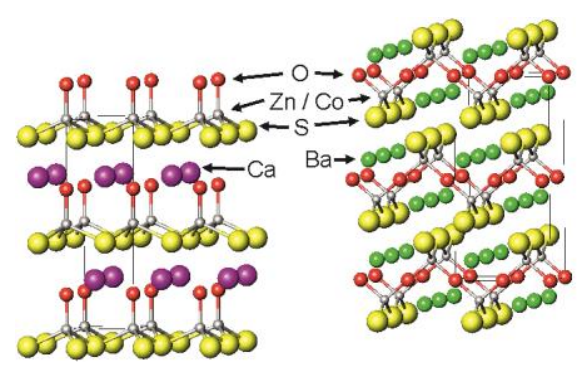

The polar antiferromagnetic oxysulfide $\mathrm{CaCoSO}$ and the related antiferromagnet $\mathrm{BaCoSO}$ are described. 